FERNANDES, P.C.; FACANALI, R.; TEIXEIRA, J.P.F.; FURLANI, P.R.; MARQUES, M.O.M. Cultivo de manjericão em hidroponia e em diferentes substratos sob ambiente protegido. Horticultura Brasileira, Brasília, v.22, n.2, p.260-264, abril-junho 2004.

\title{
Cultivo de manjericão em hidroponia e em diferentes substratos sob ambiente protegido
}

\author{
Priscila C. Fernandes'; Roselaine Facanali'; João Paulo F. Teixeira' ${ }^{1}$; Pedro R. Furlani²; Marcia Ortiz M. \\ Marques $^{1}$ \\ 'Instituto Agronômico, Centro de P\&D de Recursos Genéticos Vegetais, C. Postal 28, 13001-970 Campinas-SP; mortiz@iac.sp.gov.br; \\ ${ }^{2}$ Instituto Agronômico, Centro de P\&D de Solos e Recursos Ambientais, C. Postal 28, 13001-970 Campinas-SP
}

\section{RESUMO}

Entre as ervas aromáticas, o manjericão possui importância econômica no Brasil, sendo seu consumo tanto in natura quanto para processamento industrial, na obtenção de óleo essencial. Porém, as informações quanto à qualidade aromática dessas plantas em função das técnicas de cultivo são escassas. Avaliou-se a produtividade de duas espécies de manjericão, de folha estreita (Ocimum minimum $L$.) e de folha larga (Ocimum basilicum L.) em ambiente protegido. Os sistemas de cultivo utilizados foram: 1) hidroponia (floating); 2) substrato preparado e 3) substrato comercial. As amostragens foram realizadas durante o período de florescimento das plantas. O experimento foi realizado em Campinas (SP), em casa de vegetação com controle de umidade e iluminação naturais, utilizando-se o delineamento inteiramente casualizado, com três repetições. Foram avaliados também o rendimento e composição química dos óleos essenciais das plantas. O sistema hidropônico apresentou a maior produtividade de massa verde, aproximadamente $44 \%$ superior aos outros dois tipos de cultivo, para ambas as espécies estudadas. Não houve diferença significativa entre as formas de cultivo quanto ao rendimento e composição química dos óleos essenciais dentro da mesma espécie. Quanto à produção de óleo essencial, as plantas de manjericão de folha estreita apresentaram rendimento aproximadamente $10 \%$ mais elevado em relação às de folha larga, além de diferenças significativas na composição dos óleos essenciais entre as espécies.

Palavras-chave: Ocimum minimum L., Ocimum basilicum L., ambiente protegido, hidroponia, substratos, óleos essenciais.

\begin{abstract}
Culture of basil in substrata and hydroponic systems under protected environment

Among the aromatical herbs, basil is of great economic importance in Brazil. This species is used as fresh herb and also for essential oil extraction. To evaluate the productivity of basil in protected environment, two species, Ocimum minimum L. (narrow leaf basil) and Ocimum basilicum L. (wide leaf basil) were cultivated under greenhouse conditions, using a hydroponic system and two types of substrata. The experiment was carried out in a completely randomized design with three repetitions. Samples were obtained during the flowering period. The yield and the chemical composition of the essential oils were evaluated. The highest yield of green mass from the two species, was obtained in the hydroponic system, with higher productivity (aprox. 44\%) than the plants cultivated in prepared commercial substratum. Plants of Ocimum minimum L. produced more essential oil (aprox. 10\%) than plants of Ocimum basilicum L. Significant differences were found in the chemical composition of essential oil between species. No differences were observed in the chemical composition of essential oil, comparing the three cultivation systems.
\end{abstract}

Keywords: Ocimum minimum L., Ocimum basilicum L., protected environment, hydroponics system, substrates, essential oils.

\section{(Recebido para publicação em 6 de agosto de 2003 e aceito em 6 de janeiro de 2004)}

\begin{abstract}
A olericultura brasileira tornou-se um importante "agribusiness", estimado em mais de US\$ 4 bilhões no seu valor agregado (Costa, 2000). O sistema de ambiente protegido tem permitido grande aumento da produção de hortaliças por possibilitar a produção em períodos de entressafra. No campo é limitado o manejo dos fatores ambientais, consistindo fundamentalmente em ajustar as culturas ao ambiente, por meio da determinação de épocas de cultivo, da eficiência do uso da água e da busca de resistência a fatores adversos como ventos, excesso ou escassez de chuvas, dentre outros (Andriolo, 2000).

Apesar do crescimento em importância do cultivo hidropônico, poucos es-
\end{abstract}

tudos têm sido desenvolvidos para comparar a produção das espécies produzidas em ambiente protegido com as produzidas em cultivo tradicional, especialmente quando se trata de plantas aromáticas. Também são pouco estudadas as características aromáticas dessas plantas relacionadas às formas de cultivo.

No Brasil, o manjericão é cultivado principalmente por pequenos produtores rurais para a comercialização da planta como condimento (Teixeira et al., 2002). Além do uso in natura o manjericão é muito utilizado para a obtenção de óleo essencial, importante na indústria de perfumaria e na aromatização de alimentos e bebidas (Marotti et al.,1996). O óleo essencial de manjericão também apresenta propriedades inseticidas e repelentes (Umerie et al., 1998). Na região do Mediterrâneo a erva é plantada nos beirais das janelas para repelir mosquitos e moscas domésticas (Duke, 1991). Têm sido demonstradas também, atividades antimicrobianas, além de seu uso na conservação de grãos (Montes-Belmont e Carvajal, 1998).

O presente trabalho teve como objetivo avaliar a produtividade de biomassa, o rendimento e a composição química de óleo essencial, em manjericão de folha larga (Ocimum basilicum L.) e no manjericão de folha estreita (Ocimum minimum L.), cultivados em 
ambiente protegido utilizando-se hidroponia, substrato preparado e substrato comercial.

\section{MATERIAL E MÉTODOS}

Sementes de manjericão, de folha estreita e de folha larga foram postas para germinar em espuma fenólica, em ambiente protegido, não controlado. Das plântulas obtidas, 180 foram transplantadas, 20 plantas por parcela sendo 10 de cada espécie, para seus locais definitivos. O delineamento experimental utilizado foi inteiramente casualizado, com 3 tratamentos, consistindo em três sistemas de cultivo (hidroponia, substrato comercial e substrato preparado) e três repetições, totalizando nove parcelas.

Para o cultivo hidropônico (Hi), em sistema floating, utilizou-se a solução nutritiva recomendada por Furlani (1996), com condutividade elétrica de 1,60-1,65 mS/cm e a composição química, em mg/L: N-NO 3 (223); $\mathrm{N}^{-N_{4}}$ (26,5); P (39); K (294); Ca (190); Mg (24); S (33); B (0,18); Cu (0,025); Zn $(0,10) ; \operatorname{Mn}(0,325) ; \operatorname{Mo}(0,05)$ e Fe $(2,5)$. Para os outros tratamentos utilizou-se substrato comercial (Sc) organo-mineral para olericultura Plantmax Hortaliças HA, da Empresa Eucatex Agro; e substrato preparado ( $\mathrm{Sp}$ ) composto de uma parte de subsolo, duas partes de esterco enriquecido com NPK 4-14-8 e seis partes de areia grossa.

Nesses tratamentos utilizaram-se vasos plásticos de 5 litros sendo as plantas irrigadas diariamente durante o seu desenvolvimento, e uma vez por semana, a partir da segunda semana após o transplante. Em ambos os tratamentos as plantas eram irrigadas com a solução nutritiva utilizada no tratamento hidropônico.

O experimento foi instalado em ambiente protegido utilizando-se estufa agrícola com sistema autônomo de manutenção de umidade (umidificador e exaustão de ar quente) e iluminação natural. As plantas foram mantidas em bancadas de aproximadamente $1 \mathrm{~m}$ de altura.

A primeira amostragem foi feita quando as plantas atingiram de $10-20 \%$ de florescimento, e a segunda quando todos os tratamentos apresentavam per- centagem igual ou superior a $15 \%$ de plantas florescidas. Pesou-se a parte aérea de 10 plantas por tratamento para avaliação de matéria verde. As folhas das plantas foram manualmente separadas do caule e pesadas. Para obtenção da matéria seca o material vegetal foi mantido em estufa com circulação forçada de ar, a $60^{\circ} \mathrm{C}$, até peso constante.

As folhas foram secas em bandejas mantidas em temperatura ambiente para extração dos óleos essenciais. Empregou-se para tanto, destilação por arraste a vapor em equipamento Moritz por uma hora e meia, utilizando cerca de 50 $\mathrm{g}$ de folhas secas.

A composição química dos óleos essenciais foi conduzida em cromatógrafo a gás acoplado a espectrômetro de massas (CG-EM, Shimadzu - QP5000), operando por impacto de elétrons $(70 \mathrm{eV})$, dotado de coluna capilar DB-5 (30 $\mathrm{mx} 0,25 \mathrm{mmx} 0,25 \mu \mathrm{m})$, hélio como gás de arraste $(1,0 \mathrm{ml} / \mathrm{min})$, injetor a $240^{\circ} \mathrm{C}$ e detector a $230^{\circ} \mathrm{C}$. Os óleos essenciais foram solubilizados em acetato de etila (PA, Merck, $5 \mathrm{mg}$ óleo/1ml solvente), injetado $1 \mathrm{ml}$ de solução, split:1/20 e o seguinte programa de temperatura: $60^{\circ} \mathrm{C}-280^{\circ} \mathrm{C}$ a $3^{\circ} \mathrm{C} / \mathrm{min}$.

As substâncias foram identificadas pela comparação dos seus espectros de massas com o banco de dados do CGEM (Nist 62.lib), literatura (McLafferty e Stauffer, 1989) e índice de retenção (Adams, 1995).

Os resultados experimentais foram analisados estatisticamente utilizando o método ANOVA, MINITAB Statistical Software, 13.0 demo.

\section{RESULTADOS E DISCUSSÃO}

O manjericão de folha estreita (Ocimum minimum) apresentou-se 12 dias mais precoce, para a característica início de florescimento, que o manjericão de folha larga (Ocimum basilicum), independentemente do sistema de cultivo utilizado. Por esse motivo a primeira colheita foi efetuada em datas diferentes, trinta dias após o transplante (30DAT) para manjericão folha estreita e quarenta e dois dias após o transplante (42DAT) para o de folha larga, para que as plantas fossem observadas e ana- lisadas segundo seu padrão de florescimento. Verificou-se também, ao contrário do observado para a espécie com folha estreita, que o florescimento da espécie folha larga, além de ser mais tardio, ocorria em ritmo mais lento. Quando as plantas de folhas estreitas apresentavam-se, em média, 20\% florescidas (30DAT), as de folha larga apresentavam percentual médio de $10 \%$ (42DAT).

O início de florescimento deu-se aos 30 a 42 dias, para as duas espécies estudadas, concordando com o relatado por Furlan (2000) para o gênero Ocimum.

$\mathrm{Na}$ segunda colheita, aos 54 dias após o transplante (54 DAT) para as duas espécies, o manjericão de folha estreita e de folha larga apresentavam, respectivamente, para os tratamentos hidroponia, substrato preparado e substrato comercial, $53 \% ; 60 \% ; 30 \%$ e $15 \% ; 80 \% ; 65 \%$ das plantas florescidas.

A produtividade das duas espécies de manjericão nos três sistemas de cultivo foi avaliada pela produção de massa verde. As plantas de manjericão de folha estreita apresentaram em geral menor massa verde acumulada (483; 227 e 246 g/planta) do que a de manjericão de folha larga $(452 ; 329$ e 347 g/ planta), para hidroponia, substrato preparado e substrato comercial, respectivamente. Dados que concordam com o relatado por Teixeira et al (2002).

Plantas em hidroponia apresentaram maior massa verde total, seguida pelo tratamento onde se utilizou o cultivo em substrato comercial e em substrato preparado. A época de colheita foi relevante para a produção de massa verde para manjericão de folha estreita. Essa espécie aumentou a produção de massa verde (g/planta) durante todo o período estudado: 172 (30DAT) e 311 (54DAT) para hidroponia; 64 (30DAT) e 163 (54DAT) para substrato preparado e 62 (30DAT) e 184 (54DAT) para substrato comercial. Verificou-se, também, que as plantas de folha estreita, ao contrário das de folha larga, continuaram a ramificarse após o início do florescimento, o que possivelmente ocasionou diferença significativa na quantidade de massa verde acumulada.

Em plantas de manjericão de folha estreita, houve uma diferença significati- 
Tabela 1. Variação nos percentuais de matéria seca em plantas de manjericão de folha estreita e de folha larga. Campinas, IAC, 2000.

\begin{tabular}{lcccccc}
\hline \multirow{2}{*}{ Tratamento } & \multicolumn{3}{c}{ Folha estreita } & \multicolumn{3}{c}{ Folha larga } \\
\cline { 2 - 7 } & 30DAT ${ }^{1,2}$ & 54DAT & Média & 42DAT & 54DAT & Média \\
\hline $\mathrm{Hi}^{3}$ & 19 & 12 & $15 \mathrm{~B}$ & 19 & 12 & $15 \mathrm{a}$ \\
$\mathrm{Sp}$ & 33 & 13 & $23 \mathrm{~A}$ & 15 & 12 & $13 \mathrm{a}$ \\
$\mathrm{Sc}$ & 26 & 12 & $19 \mathrm{~A}$ & 15 & 13 & $14 \mathrm{~A}$ \\
\hline Média & $26,0 \mathrm{a}$ & $12,3 \mathrm{~b}$ & & $16,3 \mathrm{a}$ & $12,3 \mathrm{a}$ & \\
\hline
\end{tabular}

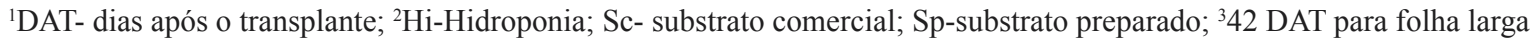

Letras diferentes, minúsculas dentro da mesma espécie e linha e maiúsculas dentro da mesma espécie e coluna, indicam diferenças significativas $(\mathrm{p} \leq 5 \%)$

Tabela 2. Massa seca de plantas (médias) de duas espécies de manjericão em função da época de colheita, em g/planta. Campinas, IAC, 2000.

\begin{tabular}{lcccccc}
\hline \multirow{2}{*}{ Tratamento } & \multicolumn{3}{c}{ Folha estreita } & \multicolumn{3}{c}{ Folha larga } \\
\cline { 2 - 7 } & 30DAT ${ }^{1,2}$ & 54DAT & Média & 42DAT & 54DAT & Média \\
\hline $\mathrm{Hi}^{3}$ & 33 & 37 & $35 \mathrm{a}$ & 45 & 26 & $36 \mathrm{a}$ \\
$\mathrm{Sp}$ & 21 & 21 & $21 \mathrm{~b}$ & 23 & 21 & $22 \mathrm{~b}$ \\
$\mathrm{Sc}$ & 16 & 22 & $19 \mathrm{~b}$ & 22 & 26 & $24 \mathrm{~b}$ \\
\hline
\end{tabular}

${ }^{1}$ DAT- dias após o transplante; ${ }^{2}$ Hi-Hidroponia; Sc- substrato comercial; Sp-substrato preparado; ${ }^{3} 42$ DAT para folha larga;

Letras diferentes dentro da mesma espécie indicam diferenças significativas $(\mathrm{p} \leq 5 \%)$

Tabela 3. Rendimento em óleos essenciais ${ }^{1}$ de folhas em duas espécies de manjericão, cultivadas em ambiente protegido. Campinas, IAC, 2000.

\begin{tabular}{lcccccc}
\hline \multirow{2}{*}{ Tratamento } & \multicolumn{3}{c}{ Folha estreita } & \multicolumn{3}{c}{ Folha larga } \\
\cline { 2 - 7 } & 30DAT ${ }^{1,2}$ & 54DAT & Média & 42DAT & 54DAT & Média \\
\hline $\mathrm{Hi}^{4}$ & 0,10 & 0,23 & 0,16 & 0,12 & 0,10 & 0,11 \\
$\mathrm{Sp}$ & 0,14 & 0,34 & 0,24 & 0,10 & 0,10 & 0,10 \\
$\mathrm{Sc}$ & 0,24 & 0,26 & 0,25 & 0,16 & 0,15 & 0,15 \\
\hline Média & $0,16 \mathrm{~b}$ & $0,27 \mathrm{a}$ & $0,22 \mathrm{~A}$ & $0,13 \mathrm{a}$ & $0,12 \mathrm{a}$ & $0,11 \mathrm{~B}$ \\
\hline
\end{tabular}

1\% óleo/ms de folhas; ${ }^{2} \mathrm{DAT}=$ dias após o transplante; ${ }^{3} 42 \mathrm{DAT}$ para folha larga; ${ }^{4} \mathrm{Hi}=$ Hidroponia; $\mathrm{Sc}=$ substrato comercial; $\mathrm{Sp}=$ substrato preparado

Letras diferentes, minúsculas para mesma espécie e linha e maiúscula entre espécies,indicam diferenças significativas ( $\mathrm{p} \leq 5 \%$ )

va de massa seca das plantas por efeito da forma de cultivo, na primeira colheita (30 DAT), como mostra a Tabela 1. O manjericão de folha estreita cultivado em substrato preparado e em substrato comercial apresentou na primeira colheita maior percentual de matéria seca que as plantas hidropônicas. Na segunda colheita esse fato não foi observado.

No manjericão de folha larga não houve alteração significativa no percentual de massa seca nos diferentes tipos de cultivo e época de colheita, inferindo-se, portanto, que as diferenças de massa verde observadas, para o tratamento hidropônico, são devidas ao teor de água acumulado nos tecidos.

Para as duas espécies estudadas o percentual médio de massa seca na pri- meira colheita foi superior à da segunda colheita (54DAT), sendo essa diferença significativa apenas para a espécie de folha estreita.

O acúmulo médio de massa seca por planta, independente da espécie estudada, foi maior para plantas de cultivo hidropônico (Tabela 2). Não houve diferença significativa entre a massa seca por planta entre o manjericão folha estreita e o folha larga, diferentemente do encontrado por Teixeira et al (2002) que estudou essas mesmas espécies utilizando cultivo hidropônico (nutrient film technique).

O tipo de cultivo não influenciou significativamente o rendimento de óleos essenciais nas duas espécies estudadas (tabela 3). Houve, porém, dife- rença significativa no rendimento dos óleos essenciais entre o manjericão de folha estreita e o de folha larga. Para a espécie de folha estreita os maiores rendimentos ocorreram na segunda colheita (54 DAT). O manjericão de folha estreita apresentou, em geral, rendimentos maiores que os de folha larga nos três sistemas de cultivo. O mesmo foi observado por Teixeira et al (2002) para essas duas espécies cultivadas em hidroponia.

Não foram observadas interações significativas entre os tratamentos e espécies, nas duas épocas de colheita, com a biomassa produzida (massa verde total e massa seca) e rendimento em óleo essencial.

A composição química dos óleos essenciais das duas espécies de manje- 
Tabela 4. Composição química dos óleos essenciais (\%) de folhas de manjericão folha estreita e folha larga, cultivo em ambiente protegido. Campinas, IAC, 2000.

\begin{tabular}{|c|c|c|c|c|c|c|c|c|c|c|c|}
\hline \multirow{3}{*}{ Composição química } & \multicolumn{6}{|c|}{ folha estreita } & \multicolumn{5}{|c|}{ folha larga } \\
\hline & \multicolumn{3}{|c|}{30 DAT $^{1}$} & \multicolumn{3}{|c|}{54 DAT } & \multicolumn{2}{|c|}{$42 \mathrm{DAT}^{2}$} & \multicolumn{3}{|c|}{54 DAT } \\
\hline & $\mathrm{Hi}^{3}$ & $\mathrm{Sp}$ & $\mathrm{Sc}$ & $\mathrm{Hi}$ & Sp & Sc & $\mathrm{Hi}$ & Sc & $\mathrm{Hi}$ & Sp & Sc \\
\hline a-pineno & 0,2 & 0,3 & 0,4 & 0,1 & 0,2 & 0,5 & 0,5 & - & 0,3 & 0,5 & 0,5 \\
\hline Sabineno & 0,3 & 0,3 & 0,4 & 0,1 & 0,2 & 0,4 & 0,6 & 0,3 & $\operatorname{tr}$ & 0,6 & 0,7 \\
\hline b-pineno & 0,6 & 0,7 & 1,0 & 0,4 & 0,5 & 0,9 & 1,5 & 0,4 & 0,3 & 1,3 & 1,4 \\
\hline Mirceno & 1,0 & 1,3 & 1,4 & 1,0 & 0,7 & 1,5 & 2,0 & 0,9 & 0,4 & 1,6 & 1,7 \\
\hline Limoneno & 0,4 & 0,5 & 0,5 & 0,6 & 0,4 & 0,9 & 1,0 & 0,2 & 0,5 & 0,7 & 0,8 \\
\hline 1,8-cineol & 3,4 & 5,0 & 4,6 & 2,7 & 4,6 & 4,9 & 5,3 & 2,9 & 1,8 & 6,3 & 4,6 \\
\hline Cis-ocimeno & 0,3 & 0,6 & 0,8 & 0,3 & 0,3 & 1,0 & 1,8 & 0,6 & 0,3 & 1,9 & 1,8 \\
\hline Trans-4-tujanol & 0,2 & 0,4 & $\operatorname{tr}$ & - & $\operatorname{tr}$ & - & - & - & $\operatorname{tr}$ & $\operatorname{tr}$ & - \\
\hline Linalol & 53,0 & 51,2 & 45,5 & 54,3 & 59,8 & 44,3 & 36,6 & 37,4 & 22,7 & 33,4 & 25,1 \\
\hline Terpin-4-ol & 0,5 & 1,0 & 0,4 & 0,3 & 0,5 & 0,5 & - & - & $\operatorname{tr}$ & $\operatorname{tr}$ & $\operatorname{tr}$ \\
\hline a-terpinol & 0,4 & 0,4 & 0,4 & 0,3 & 0,3 & 0,5 & 0,4 & - & 0,4 & 0,6 & 0,6 \\
\hline Acetato de bornila & 1,1 & 1,2 & 1,1 & 1,4 & 1,2 & 2,8 & 1,9 & 3,8 & 3,6 & 3,4 & 4,2 \\
\hline Eugenol & 1,2 & 1,5 & 2,4 & 1,2 & 0,8 & 2,0 & 2,0 & $\operatorname{tr}$ & 0,8 & 2,2 & 2,0 \\
\hline a-copaeno & 0,4 & - & 0,5 & 0,4 & 0,4 & 0,5 & $\operatorname{tr}$ & $\operatorname{tr}$ & 0,6 & 0,4 & 0,4 \\
\hline Acetato de geranila & 0,4 & 0,4 & 0,3 & $\operatorname{tr}$ & 0,5 & $\operatorname{tr}$ & - & - & $\operatorname{tr}$ & - & $\operatorname{tr}$ \\
\hline b-bourboneno & $\operatorname{tr}$ & $\operatorname{tr}$ & $\operatorname{tr}$ & $\operatorname{tr}$ & - & - & - & - & 0,4 & $\operatorname{tr}$ & $\operatorname{tr}$ \\
\hline b-cubebeno & 0,3 & $\operatorname{tr}$ & 0,5 & 0,2 & 0,2 & 0,4 & - & - & $\operatorname{tr}$ & 0,3 & 0,4 \\
\hline b-elemeno & 1,4 & 1,7 & 2,0 & 2,3 & 3,0 & 3,1 & 1,8 & 1,9 & 5,5 & 2,0 & 3,9 \\
\hline Metil eugenol & 2,1 & 1,9 & 1,7 & $\operatorname{tr}$ & $\operatorname{tr}$ & - & 0,6 & - & $\operatorname{tr}$ & - & 0,5 \\
\hline a-cis-bergamoteno & - & $\operatorname{tr}$ & $\operatorname{tr}$ & - & $\operatorname{tr}$ & - & $\operatorname{tr}$ & - & $\operatorname{tr}$ & - & $\operatorname{tr}$ \\
\hline Trans-cariofileno & - & $\operatorname{tr}$ & $\operatorname{tr}$ & $\operatorname{tr}$ & - & $\operatorname{tr}$ & $\operatorname{tr}$ & - & 0,4 & - & $\operatorname{tr}$ \\
\hline a-trans-bergamoteno & 6,7 & 6,6 & 6,6 & 9,5 & 7,5 & 7,5 & 14,4 & 19,7 & 19,0 & 14,8 & 13,1 \\
\hline a-guaieno & 1,0 & 1,0 & 1,2 & 1,2 & 1,5 & 1,5 & 1,0 & 1,2 & 0,9 & 0,7 & 0,9 \\
\hline b-cis-farneseno & $\operatorname{tr}$ & $\operatorname{tr}$ & $\operatorname{tr}$ & - & $\operatorname{tr}$ & 0,2 & - & - & 0,3 & $\operatorname{tr}$ & $\operatorname{tr}$ \\
\hline a-humuleno & 0,7 & 0,6 & 0,9 & 0,6 & 1,0 & 0,7 & 0,7 & 0,6 & $\operatorname{tr}$ & 0,8 & 1,3 \\
\hline b-trans-farneseno & 3,1 & 2,0 & 2,4 & 1,8 & 0,4 & 0,5 & 3,6 & 2,0 & 3,9 & 1,7 & 1,9 \\
\hline Cis-murola-4(14),5 dieno & 0,7 & 0,3 & 0,8 & 0,7 & 0,7 & 0,8 & 0,7 & 0,7 & 1,2 & 0,8 & 1,1 \\
\hline Germacreno-D & 4,6 & 5,6 & 6,3 & 5,7 & 6,6 & 6,8 & 5,3 & 6,9 & 8,1 & 7,8 & 9,1 \\
\hline b-selineno & 0,4 & $\operatorname{tr}$ & 0,4 & 0,6 & - & 0,5 & 1,0 & 1,3 & 1,4 & 1,1 & $\operatorname{tr}$ \\
\hline Biciclogermacreno & 1,1 & 1,1 & 1,6 & 0,8 & 1,0 & 1,1 & 1,3 & 1,1 & 1,0 & 1,0 & 1,5 \\
\hline b-trans-guaieno & $\operatorname{tr}$ & $\operatorname{tr}$ & $\operatorname{tr}$ & $\operatorname{tr}$ & $\operatorname{tr}$ & $\operatorname{tr}$ & $\operatorname{tr}$ & - & $\operatorname{tr}$ & $\operatorname{tr}$ & $\operatorname{tr}$ \\
\hline a-bulneseno & 2,2 & 3,0 & 2,9 & 3,2 & 4,1 & 3,9 & 2,9 & 3,0 & 3,0 & 2,2 & 4,3 \\
\hline b-bisaboleno & $\operatorname{tr}$ & $\operatorname{tr}$ & $\operatorname{tr}$ & $\operatorname{tr}$ & - & $\operatorname{tr}$ & $\operatorname{tr}$ & - & 0,4 & $\operatorname{tr}$ & $\operatorname{tr}$ \\
\hline g-cadineno & 3,8 & 3,2 & 4,2 & 3,8 & 3,5 & 4,2 & 3,9 & 4,0 & 5,8 & 3,9 & 5,0 \\
\hline b-sesquifelandreno & $\operatorname{tr}$ & $\operatorname{tr}$ & $\operatorname{tr}$ & 0,3 & $\operatorname{tr}$ & 0,3 & 0,6 & 0,8 & 2,2 & 0,8 & 0,8 \\
\hline Espatulenol & $\operatorname{tr}$ & $\operatorname{tr}$ & - & - & $\operatorname{tr}$ & - & - & - & $\operatorname{tr}$ & - & - \\
\hline 1-10-di-epi-cubenol & 0,7 & 0,7 & 0,9 & 0,5 & 0,5 & 0,7 & 0,6 & 0,7 & 1,2 & 0,8 & 1,1 \\
\hline Cubenol & 5,7 & 6,0 & 6,7 & 4,0 & 3,9 & 4,9 & 5,3 & 6,0 & 8,0 & 5,6 & 7,1 \\
\hline Total identificado & 98,1 & 98,5 & 98,8 & 98,5 & 98,1 & 98,0 & 97,6 & 96,7 & 94,3 & 97,4 & 96,0 \\
\hline
\end{tabular}

${ }^{1} \mathrm{DAT}=$ dias após o transplante; ${ }^{2}$ tratamento $\mathrm{Sp}=$ óleo sofreu decomposição após extração; Hi = Hidroponia; $\mathrm{Sc}=$ substrato comercial; $\mathrm{Sp}=$ substrato preparado

ricão é apresentada na tabela 4, exceto do óleo essencial de manjericão folha larga cultivado em substrato preparado que sofreu decomposição. Foram identificadas 38 substâncias componentes dos óleos essenciais analisados.
As substâncias dos óleos essenciais de manjericão apresentaram como majoritárias o linalol, o $\alpha$-trans- 
bergamoteno, o germacreno D, o cubenol e o $\gamma$-cadineno. O linalol é a substância mais abundante nas espécies estudadas, tanto de folha estreita (44,3\% a $59,8 \%$ ) quanto no de folha larga $(22,7 \%$ a $37,4 \%)$. O $\alpha$-transbergamoteno é a segunda substância mais abundante nas duas espécies, atingindo teores mais elevados no manjericão de folha larga. Os dados obtidos para composição química dos óleos essenciais estão de acordo com os relatados por Furlan (2000) e Teixeira et al (2002) para essas espécies.

Houve diferença significativa na composição química de óleos essenciais de Ocimum minimum L. e de Ocimum basilicum L.. Não houve alteração significativa entre os óleos essenciais obtidos de plantas, de mesma espécie, em função dos sistemas de cultivo utilizados, evidenciando a regulação genética da biossíntese dessas substâncias.

Conclui-se, portanto, que para as duas espécies de manjericão estudadas, o sistema hidropônico possibilitou maior produtividade, cerca de $44 \%$, em média, a mais de massa verde, na segunda amostragem das plantas, em relação a outros meios de cultivo. Devese lembrar que todos os tratamentos eram semelhantes quanto à disponibilidade de nutrientes para as plantas, já esses eram repostos a partir de uma solução de mesma concentração.

O rendimento e a composição química dos óleos essenciais das plantas cultivadas em hidroponia, não diferiram significativamente dos obtidos de plantas cultivadas em substrato preparado e substrato comercial.

Evidencia-se, com os resultados obtidos, que o uso de sistema hidropônico é adequado à produção de manjericão para comércio da planta fresca, sem prejuízo da característica aromática conferida pelos óleos essenciais.

\section{AGRADECIMENTOS}

Os autores agradecem ao CNPq pela concessão de bolsa de iniciação científica a Priscila Correia Fernandes (proc. $\mathrm{n}^{\mathrm{o}} 101175$ 100-0).

\section{LITERATURA CITADA}

ADAMS, R.P. Identification of essential oils by gas chromatography/mass espectroscopy. Carol Stream: Allured Pub. Co., USA., 1995. 469 p.

ANDRIOLO, J.L. Fisiologia da produção de hortaliças em ambiente protegido. Horticultura Brasileira, Brasília, v.18, Suplemento, p.26-33, 2000.
COSTA, C.P. Olericultura Brasileira: passado, presente e futuro. Horticultura Brasileira, Brasília, v.18, Suplemento, p.7-14, 2000.

DUKE, J.A. Handbook of medicinal herbs. Boca Raton, 1991. p.332-334.

FURLAN, M.R. Efeito da adubação com $N$ P2O5-K2O sobre a biomassa, o rendimento e a composição do óleo essencial de Ocimum basilicum L. Cultivar Genovese. FCA-UNESP, Botucatu, 2000, 172 p. (Tese de doutorado)

FURLANI, P.R. Hidroponia, In: VAN RAIJ, B.; CANTARELLA,H; QUAGGIO,J.A.; FURLANI, A.M.C. - Recomendações de adubação e calagem para o Estado de São Paulo, 2a . Edição, Campinas, 1996, 285 p. (Boletim Técnico, IAC, ${ }^{\circ}{ }^{\circ} 100$ ) MAROTTI, M., PICCAGLIA, R., GIOVANELLI, E. Differences in essential oil composition of Basil (Ocimum basilicum L.) italian cultivars related to morfological characteristics. Journal of Agricultural Food Chemistry v.44,n.12, p.39263929, 1996.

McLAFFERTY, F.W.; STAUFFER, D.B. Registry of mass espectral data. v.1. New York: WilleyInterscience Pub., 1989. 1038 p.

MONTES-BELMONT, R.; CARVAJAL, M. Control of Aspergillus flavus in maize with plant essential oils and their components. Journal of Food Protection, v.61, n.5, p.616-619, 1998.

TEIXEIRA, J.P.F.; MARQUES, M.O.M.; FURLANI, P.R.; FACANALLI, R. Essential oil contents in two cultivars of basil cultivated on NFT-hydroponics. IN: Proceedings of the First Latin-American Symposium on the Production of Medicinal, Aromatic and Condiments Plants, Acta Horticulturae, v.569, p.203-208, 2002.

UMERIE, S.C., ANASO, H.U.; ANYASORO, L.J.C. Inseticidal potentials of Ocimum basilicum leaf extracts. Bioresource Technology, v.64, n.3, p.237-239, 1998. 